\title{
Molecular Cloning and Functional Characterization of the DELLA Gene Family in Liriodendron Hybrids
}

\author{
Yang Liu ${ }^{1,+} \mathbb{\oplus}$, Pengkai Wang ${ }^{1,2,+}$, Shan Yan ${ }^{1}$, Xin Liu ${ }^{1}$, Lu Lu $^{1}{ }^{1}$, Xinying Chen ${ }^{1}$, Ye Lu ${ }^{1}$, \\ Zhaodong Hao ${ }^{1}$, Jisen Shi ${ }^{1}{ }^{1}$ and Jinhui Chen ${ }^{1, *}$ \\ 1 Key Laboratory of Forest Genetics \& Biotechnology of Ministry of Education, \\ Co-Innovation Center for Sustainable Forestry in Southern China, Nanjing Forestry University, \\ Nanjing 210037, China; yangliunjfu@gmail.com (Y.L.); mengxiao02181@gmail.com (P.W.); \\ yanshan201111@gmail.com (S.Y.); meteorliuxin01@gmail.com (X.L.); lulunjfu@gmail.com (L.L.); \\ chenxinying0531@gmail.com (X.C.); luye@njfu.edu.cn (Y.L.); haozd@njfu.edu.cn (Z.H.); \\ jshi@njfu.edu.cn (J.S.) \\ 2 Suzhou Polytechnic Institute of Agriculture, Suzhou 215008, China \\ * Correspondence: chenjh@njfu.edu.cn; Tel.: +86-5-85428817 \\ + The Authors share senior co-authorship.
}

Received: 8 November 2020; Accepted: 16 December 2020; Published: 18 December 2020

\begin{abstract}
DELLA proteins are key components of the gibberellins signal transduction pathway that play negative roles on promoting cell elongation and plant stature. However, the mechanisms underlying DELLA mediated growth inhibition in woody plant species are poorly understood. Here, we identified two LhDELLA genes including LhSLR-like (LhSLRL) and LhRGA from Liriodendron hybrids (Liriodendron chinense (Hemsl.) Sarg. $\times$ L. tulipifera L.), which is a horticultural tree with high-quality timber. Sequence analysis showed that LhSLRL and LhRGA possessed all typical conserved domains of DELLA proteins. Phylogenetic analysis showed that LhRGA was classified as the basal branch of DELLAs from species belonging to dicots. Interestingly, LhSLRL was a sister clade of DELLAs from the most ancient plant species such as bryophytes and ferns. Gene expression analysis showed that LhSLRL and LhRGA genes had the highest expression level in the stem. In addition, overexpression of LhSLRL decreased plant height and root length, increased branching and delayed flowering in Arabidopsis thaliana, while LhRGA overexpression enlarged leaves and reduced the number and length of roots. Overexpressing LhSLRL in tobacco caused a decreased plant height and the amount of root. Taken together, these results reveal that LhDELLA genes may play an important role in plant growth and development, especially in vegetative organs. Our results provide new insights into the function of DELLA genes in a woody plant, and contribute to a potential application of manipulating DELLA genetics.
\end{abstract}

Keywords: Liriodendron hybrids; DELLA; RGA; SLRL; gibberellin

\section{Introduction}

The plant hormones gibberellins (GAs) were first identified from rice diseased with the "bakanae" fungus ("Bakanae" fungus secretes a large amount of gibberellin, which makes rice grow steep) in 1926 and were officially named gibberellins in 1938. It is well known that GA induces a wide range of vegetative and reproductive developmental processes in plants, such as seed germination, hypocotyl elongation, stem elongation, leaf expansion, flower regulation, and fruit development $[1,2]$.

Through the study of mutants defective in GA biosynthesis or signal transduction in rice (Oryza sativa cv Nipponbare) and Arabidopsis (Arabidopsis thaliana (L.) Heynh.), several GA signaling pathway components were identified [3-6]. For example GIBBERELLIN INSENSITIVE 1 (GID1), which 
acts as a hormone-sensitive lipase, can interact with DELLA proteins to modulate GA signaling [5-7]. Other GA regulatory proteins, including the F-box proteins (SLEEPY1 [SLY1] and SNEEZY (SNE)/SLY2 in Arabidopsis [8], GIBBERELLIN INSENSITIVE 2 [GID2] in rice [9]), have also been identified via various genetic and biochemical approaches.

DELLA proteins play a negative regulatory role in GA signaling and are named for their $\mathrm{N}$-terminal DELLA domain, which belongs to a larger transcription factor (TF) family-The GRAS TF families [10]. DELLA genes have been cloned from dozens of plant species, such as Arabidopsis [11], rice [3], wheat (Triticum aestivum L.) [12], rape (Brassica napus L.) [13], strawberry (Fragaria vesca L.) [14] etc., and their functions and regulatory mechanisms have been studied in depth, with more emphasis on Arabidopsis, rice, and other model plants. In the $A$. thaliana genome, five DELLA genes are encoded: RGA (Repressor of ga1-3) [15,16], GAI (GA-Insensitive) [4], RGL1 (RGA-like1) [11], RGL2 [17], and RGL3 [18].

The DELLA proteins are a set of plant-specific TFs, whose typical architecture consists of a conserved GA-response specific domain, and a highly conserved GRAS domain. The GA-response specific domain is composed of DELLA, a TVHYNP motif and a poly S/T/V region. DELLA, LEQLE, and TVHYNP motifs confer the GA signal-sensing and transcriptional coactivation capacity of this domain, which is functionally conserved across all land plants [19]; the poly $\mathrm{S} / \mathrm{T} / \mathrm{V}$ region is a regulatory motif containing a protein phosphorylation and glycosylation modification site. The GRAS domain consists of NLS, LXXLL (or LZ), VHIID, SH2, and SAW motifs. The NLS motif is a nuclear localization signal motif and plays an important role in the localization of DELLA proteins. LZ (Leucine Zipper) is a leucine motif that promotes dimer formation, transcription co-activation, and interaction with nuclear receptors. VHIID, SH2, and SAW are repressive functional motifs [20].

The DELLA protein N-terminus can sense GA signaling, which is the structural basis of DELLA protein degradation. In the A. thaliana mutants gai-1 and rga-17, the function of one DELLA protein is lost, resulting in reduced GA sensitivity. In these two mutants, GA cannot cause the degradation of DELLA through the ubiquitin-proteasome pathway, leading to plant dwarfing. Meanwhile, deletion of five amino acids from the DELLA motif at the N-terminus of the $d 8-1$ mutant DELLA protein (Zea mays L.) or mutation of the DELLALG motif to DELLALE in the Sln1d mutant (Hordeum vulgare L.), leads to a similar dwarfing phenotype [12,16,21,22]. In wheat, either an insertion of 30 amino acids into the DELLA structural domain or terminating mutations near the DELLA motif $(r h t-d 1 c$ and $R h t-b 1 b / R h t-d 1 b$ mutants, respectively) weaken the interaction of the Rht3 DELLA gene with GID1, and therefore, cause semi-dwarf and semi-dominant mutant phenotypes. In addition, single base variation in the DELLA domain resulted in no GA response and plant dwarfing [4,12]. The GRAS region at the C-terminus of DELLA proteins is responsible for the interaction with hundreds of TFs $[23,24]$. This interaction either downregulates $[25,26]$ or enhances $[27,28]$ the activity of the TFs, thereby regulating thousands of genes in different contexts [29].

The "Green Revolution" of the 1950s was made possible by beneficial mutations of GA20ox2 and DELLA proteins in rice and wheat, respectively, to achieve semi-dwarf breeding, leading to a strongly increased production of food [12]. Now, in a variety of crops, the DELLA protein expression level can be regulated using transgenics to create dwarf plants, increasing stress resistance and yield [30]. In addition, DELLA proteins play an important role in releasing seed dormancy, promoting early flowering, prolonging the flowering period, improving fruit quality, delaying plant senescence, and regulating the synthesis of secondary metabolites [17,31,32]. Therefore, the study of DELLA proteins has broad application prospects in regulating plant growth and development, and stress resistance [32,33].

The function of DELLA proteins is highly conserved among different plant species, but the number of DELLA genes and their mode of action differs across different species. In this study, we identified two LhDELLA genes, LhSLRL and LhRGA. We isolated and characterized these genes to speculate their role in Liriodendron hybrids growth and development. 


\section{Materials and Methods}

\subsection{Plant Materials and Growth Conditions}

Organs/tissues including root, stem, bud, leaf, flower, petal, stamen, and pistil from 25-year-old Liriodendron hybrids planted in the Xiashu Forest Farm of Nanjing Forestry University (32 $7^{\prime} 48^{\prime \prime} \mathrm{N}$, $119^{\circ} 13^{\prime} 12^{\prime \prime} \mathrm{E}$ ), were collected and frozen in liquid nitrogen, then stored at $-80^{\circ} \mathrm{C}$ for further analysis.

The Arabidopsis ecotype used in this study is Col-0, while the tobacco used is wild-type tobacco (Nicotiana tabacum L.). Arabidopsis seedlings were grown on 1/2 MS Culture Medium, tobacco seedlings were grown on MS medium. Adult wild-type and transgenic plants of both species were grown in a greenhouse (21 to $25^{\circ} \mathrm{C}$, 16-h-light/8-h-dark cycle with supplemental light of $\sim 300 \mu \mathrm{E} \mathrm{m}{ }^{-2} \mathrm{~s}^{-1}$, T5 $28 \mathrm{~W}$ $6400 \mathrm{~K}$ three-band linear fluorescent lamps and 60 to $80 \%$ humidity). Soil was composed of nutrient soil, vermiculite and perlite in a 5:1:1 ratio at identical dry weight per pot and was watered daily to maintain a water content of about $0.75 \mathrm{~g}$ of water per $\mathrm{g}$ of dry soil.

\subsection{Identification of DELLA Genes in Liriodendron Hybrids}

Protein sequences of the rice and Arabidopsis DELLA family were downloaded from NCBI (http: //blast.ncbi.nlm.nih.gov/), and the windows native comparison software BioEdit v.7.1 (NCSU, Raleigh, NC, USA) was used to compare the BLASTP transcript histone sequence of Liriodendron hybrids to the protein sequences of rice and Arabidopsis DELLA to obtain candidate protein sequences. In order to further verify the reliability of the candidate protein, the sequence of the candidate protein was extracted from the transcript histone file, compared to the Pfam database (https:/www.ebi.ac.uk/Tools/pfa/pfamscan/), and the genes with DELLA (Pfam 12041) and GRAS domain (Pfam 03514) were retained.

\subsection{Analysis of Gene Structure and Motif}

DNAMAN v.9.0 (Lynnon Corporation, San Ramon, CA, USA) was used to align the DELLA protein sequences of Arabidopsis and the Liriodendron hybrids. Conserved motifs in the protein sequences were verified by MEME (http://meme-suite.org/tools/meme), which provided information on their position in the protein sequence as well. TBtools v.1.0 (SCAU, Guangzhou, Guangdong, China) [34] was used to visualize amino acids conservation within motifs, as well as their distribution within each protein sequence.

\subsection{Sequence Alignment and Phylogenetic Analysis}

DELLA protein amino acid sequences were downloaded from the TAIR and NCBI databases, using Arabidopsis thaliana DELLA protein sequences as a query. Multiple sequence alignments were performed with Clustal W. The evolutionary history was inferred by using the Maximum Likelihood method and JTT matrix-based model [35]. The evolutionary history was inferred by using the Maximum Likelihood method and JTT matrix-based model. The percentage of trees in which the associated taxa clustered together is shown next to the branches. Initial tree(s) for the heuristic search were obtained automatically by applying Neighbor-Join and BioNJ algorithms to a matrix of pairwise distances estimated using a JTT model, and then selecting the topology with superior log likelihood value. The tree is drawn to scale, with branch lengths measured in the number of substitutions per site. Phylogenetic trees are drawn to scale, with branch lengths measured in the number of substitutions per site. The phylogenetic analysis involved 35 amino acid sequences (Table S1). Evolutionary analyses were conducted in MEGA X v.10.1 (Temple, Philadelphia, PA, USA) [36].

\subsection{Cloning of LhDELLAs}

Total RNA from Liriodendron hybrids leaves was extracted using plant Trizol reagent (Tiangen, Beijing, China), and cDNA was synthesized using a HisScript ${ }^{\circledR}$ III 1st Strand cDNA Synthesis Kit (Vazyme, Nanjing, China). According to the Liriodendron hybrids transcriptome, specific primers 
were designed (Table S2) to carry out PCR amplification. PCR reaction conditions were: $98^{\circ} \mathrm{C}$ pre-denaturation $2 \mathrm{~min} ; 98^{\circ} \mathrm{C} 30 \mathrm{~s}, 57^{\circ} \mathrm{C} 30 \mathrm{~s}, 72{ }^{\circ} \mathrm{C} 1 \mathrm{~min} 40 \mathrm{~s}, 35$ cycles; extension at $72{ }^{\circ} \mathrm{C}$ for $7 \mathrm{~min}$. After the PCR product was recovered, it was ligated into the pMD19-T vector (Takara Japan) to construct a recombinant plasmid to transform E.coli DH5 $\alpha$ competent cells (Tiangen, Beijing, China), and positive clones were screened for sequencing.

\subsection{Plasmid Construction and Transformation}

The pMD-18 T vectors containing LhSLRL or LhRGA genes were double digested using BamHI and SacI (NEB, MA, USA), after which the inserts were ligated into the pBI121 vector to obtain 35S:LhSLRL and 35S:LhRGA constructs. Both PCR and double enzyme digestion were used to confirm the constructed plasmid, and the correct recombinant plasmid was introduced into GV3101 and EHA105 competent cells using the freeze-thaw method. As previously reported [37,38], the flower dip method was used for Arabidopsis (Col-0) transformation, and the leaf-disc method was used for tobacco transformation. The transgenic plants have been tested by PCR and RT-PCR (Figures S4-S7). The primers are in Table S3.

\subsection{Quantitative RT-PCR}

Total RNA was extracted from different organs/tissues of the adult Liriodendron hybrids (Figure S3): root, stem, bud, leaf, flower, petal, stamen, and pistil, using a General plant total RNA extraction kit (BioTeke, Beijing, China). Genomic DNA was removed from total RNA using DNase. cDNA was synthesized using a HiScriptII1st Strand cDNA Synthesis Kit (Vazyme, Nanjing, China). qRT-PCR was performed on a LightCycler ${ }^{\circledR} 480$ (Roche, Switzerland), using the SYBR Green PCR master mix (Takara, Osaka, Japan). The Liriodendron hybrids $18 S$ rRNA gene was used as the reference gene for internal standard [39]. The primers used for qRT-PCR are listed in Table S3 (Figure S8). The relative expression value was calculated using 2(-Delta Delta C(T)) Method [40]. Three biological and three technical replicates were carried out for each gene.

\subsection{GA $A_{3}$ Treatment of T3 Arabidopsis thaliana Overexpressing LhRGA}

The seeds of wild-type Arabidopsis thaliana and the T3 homozygous plants with the LhRGA overexpression were sterilized with $1 \% \mathrm{NaClO}$, which were sown on solid 1/2MS medium for germination. Seedlings one week post germination were transferred on a new solid 1/2MS medium containing different $\mathrm{GA}_{3}$ concentrations. The $\mathrm{GA}_{3}$ concentration used in the medium was $0,0.1,0.5$, 1 , and $5 \mu \mathrm{mol} / \mathrm{L}$, respectively. Three homozygous transgenic lines were selected and 10 plants from each line were treated under each $\mathrm{GA}_{3}$ concentration, three experimental replicates for each testing, with wild-type Arabidopsis as a control. After 10 days, the root growth of transgenic plants and wild-type Arabidopsis thaliana was observed and analyzed.

\section{Results}

\subsection{Identification of DELLA Genes in Liriodendron Hybrids}

DELLAs mediate plant development by interacting with and regulating the activity of DNA-binding TFs to regulate the expression of downstream target genes [29]. First, to analyze the function of DELLA genes from Liriodendron hybrids (Liriodendron chinense (Hemsl.) Sarg. $\times$ L. tulipifera L.) [41], we used the rice and Arabidopsis DELLA protein sequences downloaded from NCBI to perform a BLASTP search against the Liriodendron hybrids transcriptome [42]. Then, we further verified whether the non-redundant DELLA candidate sequence had both the DELLA and GRAS domains using the Pfam database, resulting in a total of two DELLA members from the Liriodendron hybrids transcriptome. Predicted characteristics of putative proteins encoded by LhDELLA genes are listed (Table 1). Their length and molecular weight are 524 and 540 aa, 57.5 and $59.6 \mathrm{kDa}$, respectively. It is noteworthy that all LhDELLAs encode weakly acidic proteins as supported by the predicted isoelectric points (Table 1). According to predictions, LhDELLAs presumably localize to the nucleus (Table 1), like all DELLAs in $A$. thaliana. 
Table 1. List of putative DELLA gene family members of Liriodendron hybrids.

\begin{tabular}{|c|c|c|c|c|c|c|c|}
\hline \multirow{2}{*}{ Protein Name ${ }^{a}$} & \multicolumn{6}{|c|}{ Putative Proteins ${ }^{\mathbf{b}}$} & \multirow{2}{*}{ Protein Location } \\
\hline & Length (aa) & pI & MW (kDa) & Instability Index & Aliphatic Index & GRAVY & \\
\hline LhSLRL & 524 & 5.66 & 57.53 & 50.46 & 87.21 & -0.159 & Nuclear \\
\hline LhRGA & 540 & 5.18 & 59.61 & 39.03 & 85.13 & -0.23 & Nuclear \\
\hline
\end{tabular}

a Name refers to systematic designation to Liriodendron hybrids DELLAs in this work. ${ }^{\mathrm{b}}$ Molecular weight(kilodaltons), isoelectric point (pI), Instability index, Aliphatic index and Grand average of hydropathicity (GRAVY) of the deduced polypeptides were calculated using Protparam (https://web.expasy.org/protparam/). ${ }^{c}$ Protein location of the putative proteins was calculated using ProtComp v.9.0 (Softberry, Mount Kisco, NY, USA) (http://linux1.softberry.com/).

\subsection{Phylogenetic Analysis of LhDELLA Proteins}

To explore phylogenetic position of LhDELLAs, a phylogenetic tree was constructed with the protein sequences of LhSLRL, LhRGA, and 33 orthologues DELLAs from other species (Figure 1). The first clade comprised the DELLAs from species belonging to dicots. LhRGA was classified as the basal branch in the first clade, which is similar to the phylogenetic position of Liriodendron based on the genome sequence [42]. The second clade contained DELLAs from plants belonging to monocotyledons. Interestingly, LhSLRL together with Physcomitrella patens DELLAa, P. patens DELLAb, Selaginella kraussiana DELLA, Amborella trichopoda RHT1, Aquilegia coerulea DELLA3, Nelumbo nucifera GAI-like, and N. nucifera SLR1-like formed a clade, here named outgroup [19]. In the sequence alignment analysis, the DELLA motifs in LhSLRL in outgroup differed from those in the first clade (Figure 2). According to the most recent classification of Hernandez-Garcia et al., LhRGA may be "DELLA1" or "DELLA2" (hard to differentiate with this tree) and LhSLRL is a "DELLA3", which is the only one found in early diverging angiosperms, but also some basal eudicots have it (like Aquilegia coerulea var. pinetorum and Nelumbo nucifera Gaertn. Fruct. et Semin.) [19].

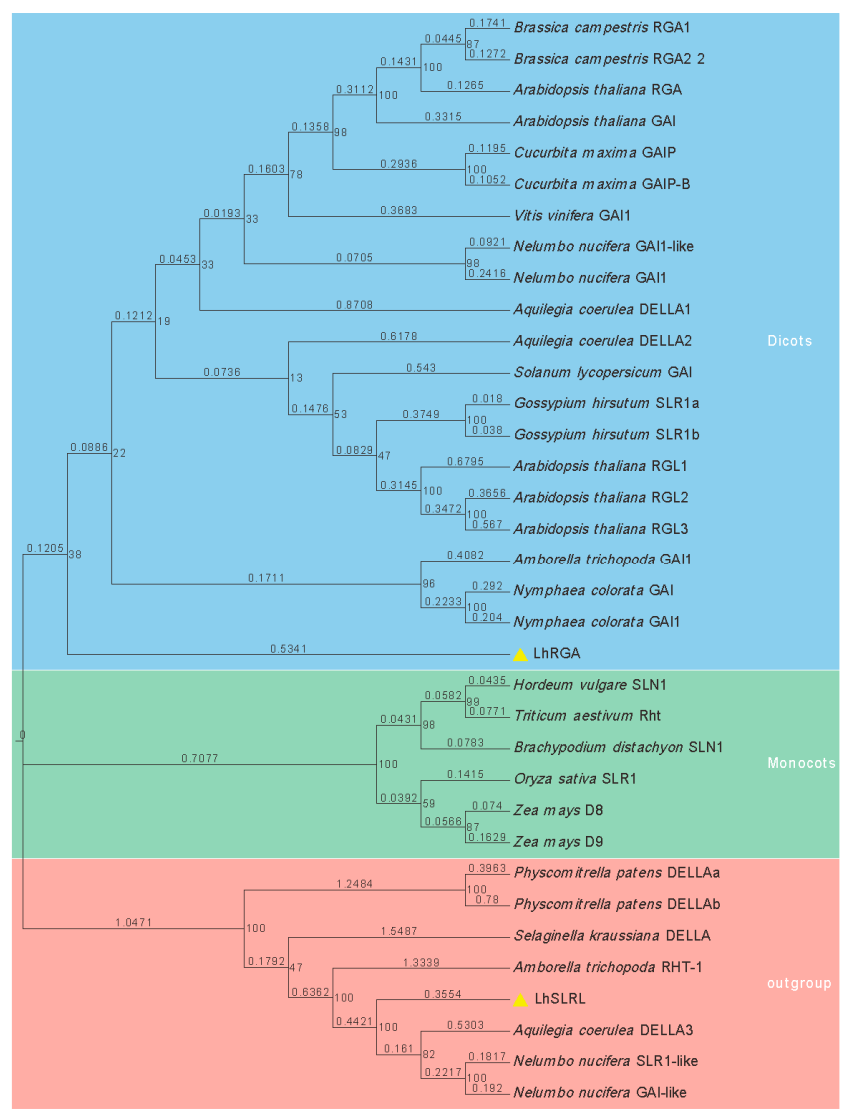

Figure 1. Phylogenetic tree of DELLA proteins from different plant species. Phylogenetic tree showing the predicted relationships between LhDELLAs and DELLA proteins of other species. Bootstrap support values are given at the nodes. This analysis involved 35 amino acid sequences. Evolutionary analyses were conducted in MEGA X. 


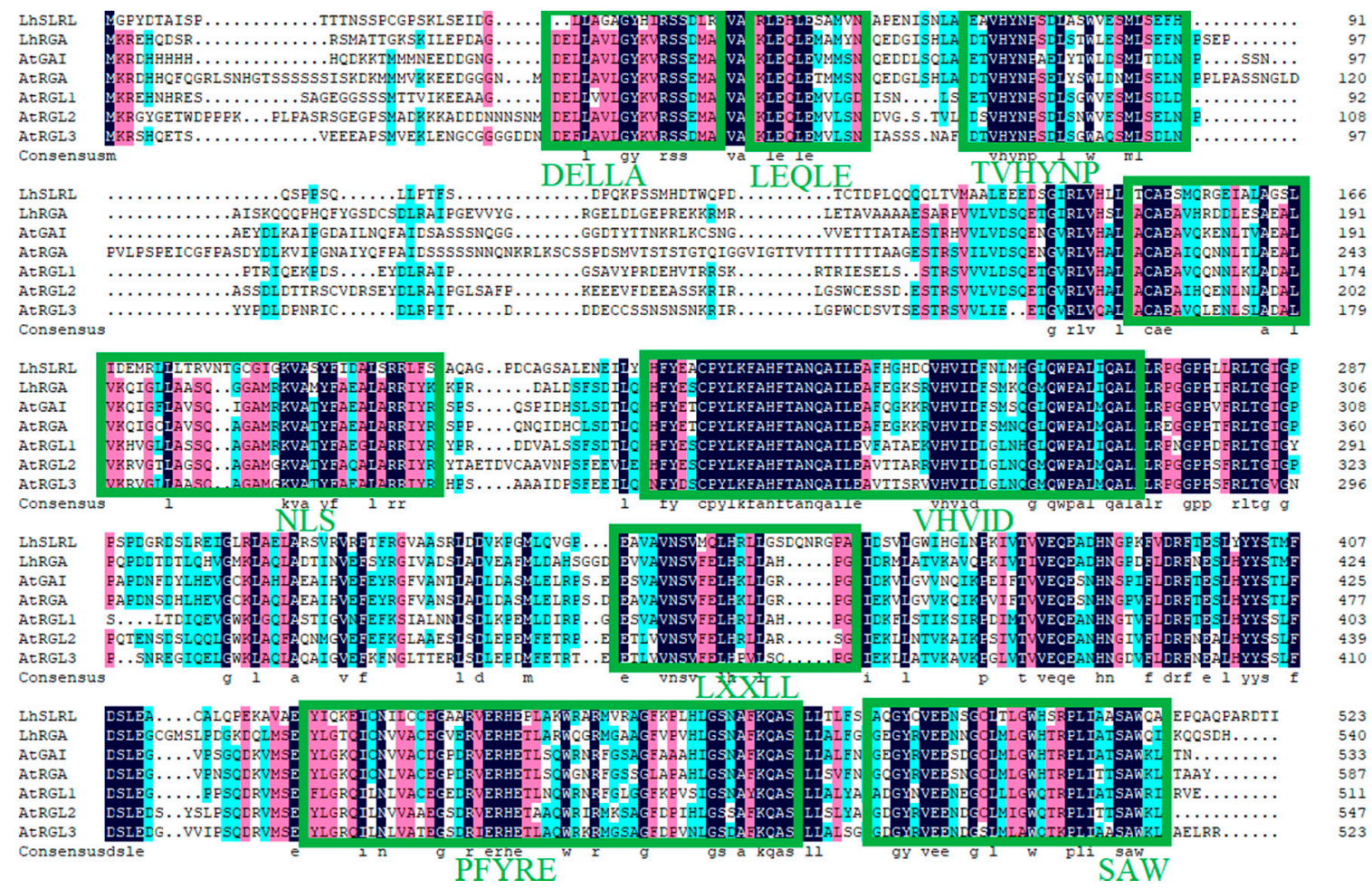

Figure 2. Sequence alignments of LhDELLAs to AtDELLAs. Amino acid sequences were aligned using DNAMAN 8. The conserved domains of the DELLA protein are framed by green lines. Identical and similar amino acid residues were shaded with black, pink, and blue, respectively.

\subsection{Structural Analysis of LhDELLA Genes}

To comprehend the conserved characteristics of Liriodendron hybrids DELLA proteins and analyze their sequence differences, we performed a multiple sequence alignment between LhDELLAs and their homologs. The results showed that LhSLRL and LhRGA proteins have $35.83 \%$ to $40.07 \%$ and $50 \%$ to $55.54 \%$ similarity with AtGAI, AtRGA, AtRGL1, AtRGL2, and AtRGL3 from Arabidopsis, respectively (Table S4). In order to better understand the sequence conservation of LhDELLAs and hypothesize about their functionality, we identified all typical and conserved amino acid motifs present across 16 DELLA proteins from nine different plant species. All selected proteins, with length varying from 511aa to 651 aa, contain more than ten motifs with a very similar arrangement (Figure 3a,b). Additionally, proteins located on the same branch of the phylogenetic tree have a similar number and arrangement of motifs. A. trichopoda GAI1, Nymphaea colorata GAIa, and N. colorata GAIb contain 17 motifs, whereas proteins from monocotyledonous plants contain motif19, which is not present in other DELLAs (Figure 3a,b). We furthermore confirmed the presence of two different motifs (DELLA, LEQLE, and TVHYNP) described previously [43] to be present in DELLA domains of Liriodendron hybrids and five different motifs (LHRI, LHRII, VHIID, PFYRE, and SAW) described previously [43] to be present in GRAS domains of Liriodendron hybrids (Figures 2 and 3b). Differences between LhRGA and AtDELLA sequences mainly lie outside the DELLA and GRAS domains, whereas the divergence of the DELLA domain sequence in LhSLRL is more substantial (Figure 2). Compared with AtDELLA sequences, LhRGA possesses motif21 but no motif14, while LhSLRL lacks motif13, motif17, motif14, motif15, motif12, and motif16 (Figure 3a). The DELLA domain of AtRGA was "DELLAVLGYKV", same as the one in LhRGA; however, the DELLA domain of LhSLRL was "DGLLAGAGYHI" (Figure 2). Together, these data indicate the divergence of extra motifs might cause changes in the characteristics of LhRGA, whereas divergence of extra motifs and variations in the DELLA domain might cause changes in the characteristics of LhSLRL. 


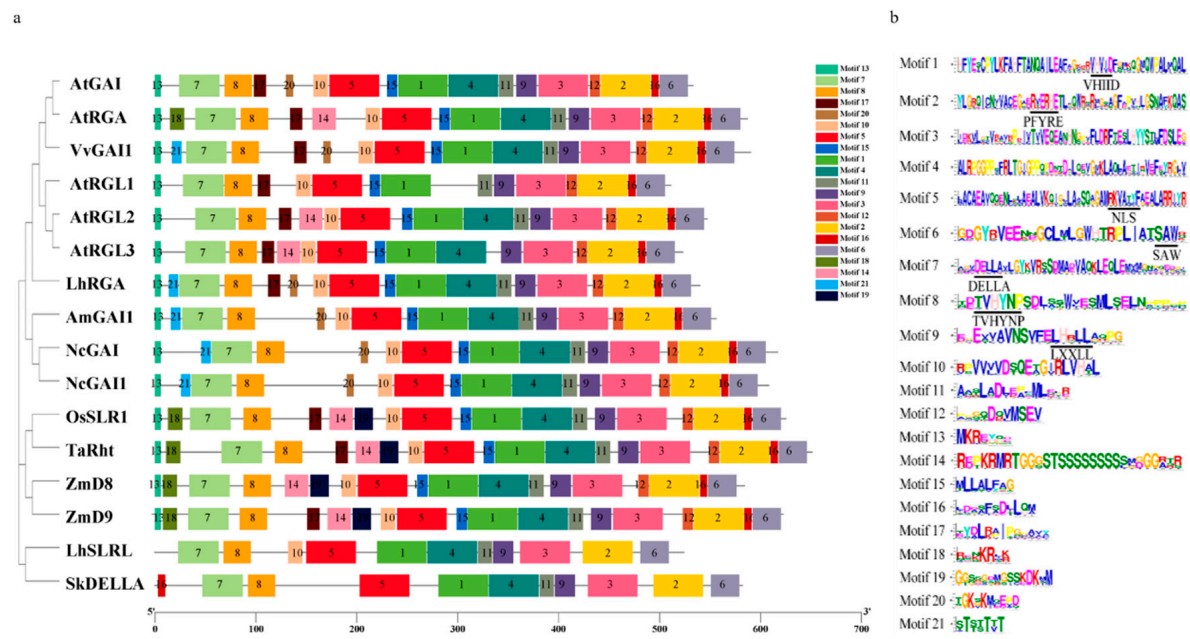

Figure 3. Structural analysis of LhDELLAs proteins. (a,b) Motifs identified (a) and their positions (b) in 16 DELLA proteins from nine different plant species. Different colors correspond to different motifs. The total height of each stack indicates the sequence conservation at that position, and the height of the symbol in each stack reflects the relative frequency of the corresponding amino acid (MEME motif search tool).

\subsection{Expression Patterns of LhDELLAs in Liriodendron Hybrids}

To further analyze LhDELLA function, we cloned the putative LhDELLA gene coding sequence using Liriodendron hybrids cDNA. We obtained a more accurate spatiotemporal distribution of $L h D E L L A$ transcripts by using quantitative real-time PCR (qRT-PCR) analysis across vegetative and reproductive organs. This analysis indicated that LhDELLAs are expressed across almost all organs/tissues, including root, stem, bud, leaf, flower, petal, stamen, and pistil, yet with largely varying expression levels. In vegetative organs, the LhSLRL and LhRGA have the highest expression level in stem, and the lowest level in root (Figure 4). However, in reproductive organs, the expression levels of $L h S L R L$ and $L h R G A$ genes are the highest level in petal, and the lowest in stamen. qPCR analysis revealed their unique spatial expression patterns in vegetative and reproductive organs of Liriodendron hybrids, indicating their potential functional diversification.

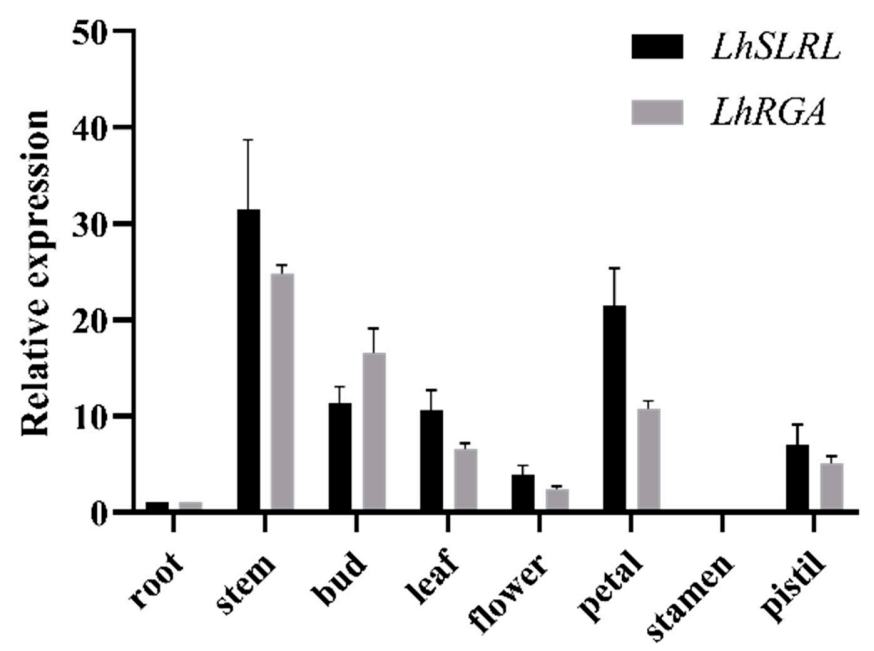

Figure 4. Expression profile of $L h D E L L A s$ in Liriodendron hybrids. Quantitative real time PCR analysis of LhDELLAs in the following organs/tissues from Liriodendron hybrids: root, stem, bud, leaf, flower, petal, stamen, and pistil. The Liriodendron hybrids $18 S$ gene was used as a reference gene. Error bars represent \pm SD from three biological repeats. 


\subsection{LhDELLA Overexpression Alters Arabidopsis Development}

To further investigate Liriodendron hybrids DELLAs functionality, we generated Arabidopsis transgenic plants overexpressing LhDELLAs under the control of the CaMV $35 S$ promoter. In Arabidopsis LhSLRL-overexpressing lines, the flowering date changed significantly, with transgenic plants flowering three-six days later than wild-type plants (Figure $5 \mathrm{a}, \mathrm{b}$ ). Flowering time showed no significant difference among four LhSLRL-overexpressing lines (Figure 5b). Furthermore, LhSLRL overexpression promoted stem development and caused branching and dwarf-like growth in transgenic plants (Figure $5 \mathrm{c}-\mathrm{e}$ ). Likewise, in Arabidopsis, the root length of LhSLRL overexpressing plants was shortened compared to wild-type plants (Figure $5 f, g$ ). We also observed these similar phenotypes in tobacco overexpressing LhSLRL (Figure S1).
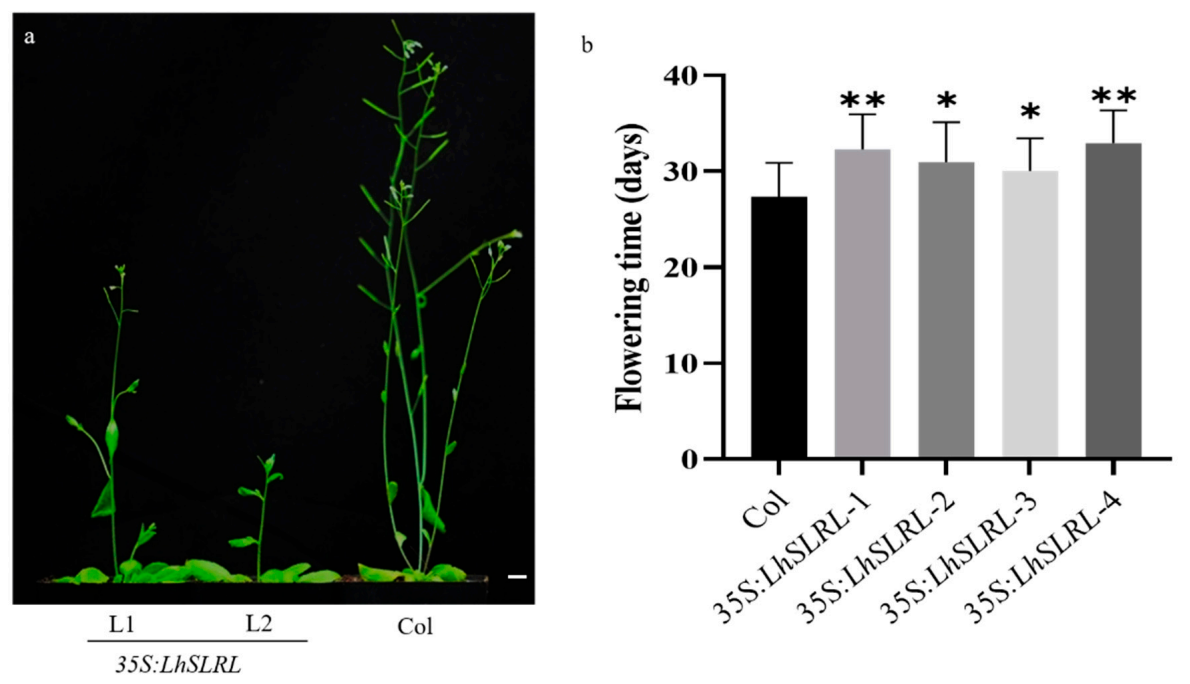

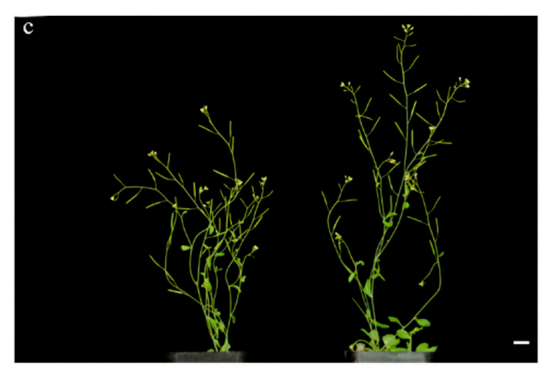

35S:LhSLRL

$\mathrm{Col}$

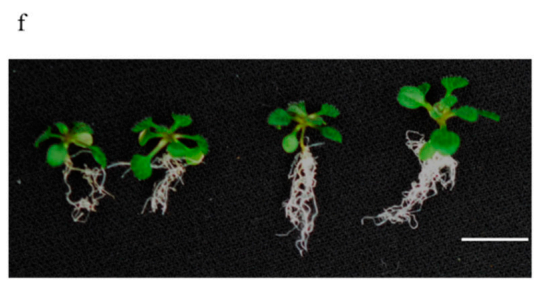

35S:LhSLRL

$\mathrm{Col}$
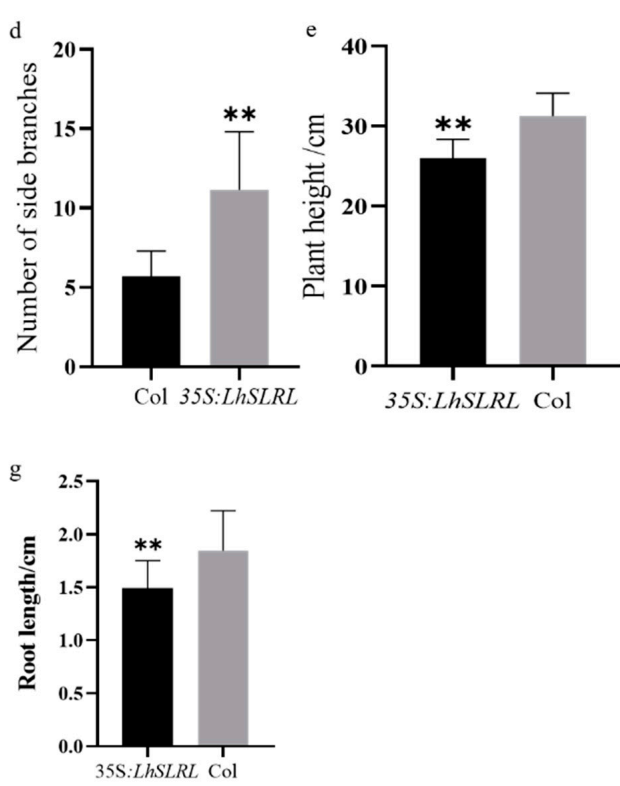

Figure 5. Morphological phenotypes of Arabidopsis overexpressing LhSLRL. (a) Phenotypes of 40-day-old wild-type and transgenic plants. (b) Measurement of flowering time in the wild-type and transgenic lines (a). (c) Phenotypes of 55-day-old wild-type and transgenic plants. (d) Number of side branches in the wild-type and transgenic lines (c). (e) Measurement of plant height branches in the wild-type and transgenic lines (c). (f) Phenotypes of 15-day-old wild-type and transgenic plants. (g) Measurement of root length in the wild-type and transgenic lines (f). Error bars: \pm SD. ${ }^{*} p<0.05 ;{ }^{* *} p<0.01$. Scale bars: $1 \mathrm{~cm}(\mathbf{a}, \mathbf{c}, \mathbf{f})$. 
Secondly, overexpression of LhRGA in Arabidopsis caused changes in plant height, flowering time, and number of side branches, but no significant difference was observed compared with the wild-type (Figure 6). However, rosette leaf length significantly increased in transgenic plants, ranging from an increase of 0.22 to $0.94 \mathrm{~cm}$ among different transgenic lines (Figure 7a,b). In addition, we found that $27 \%$ of all adult transgenic plants started to show strongly darkened green leaves (Figure S2).

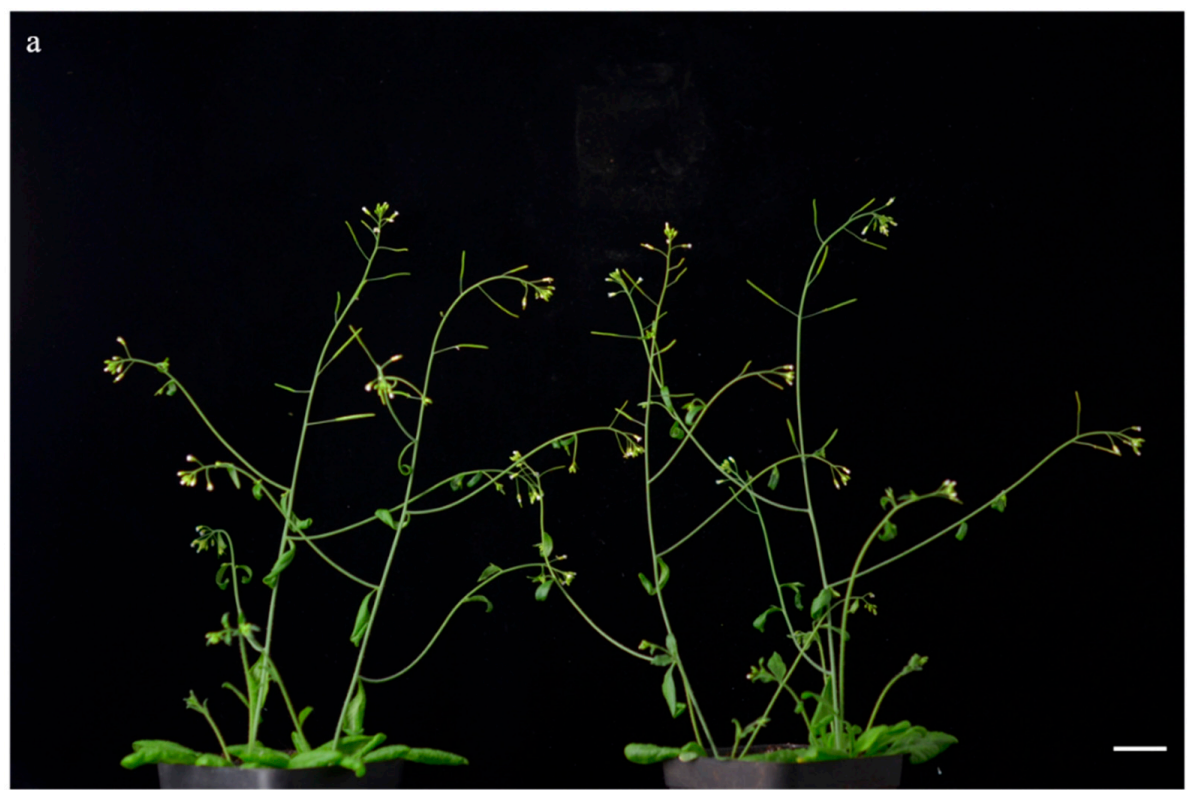

35S:LhRGA

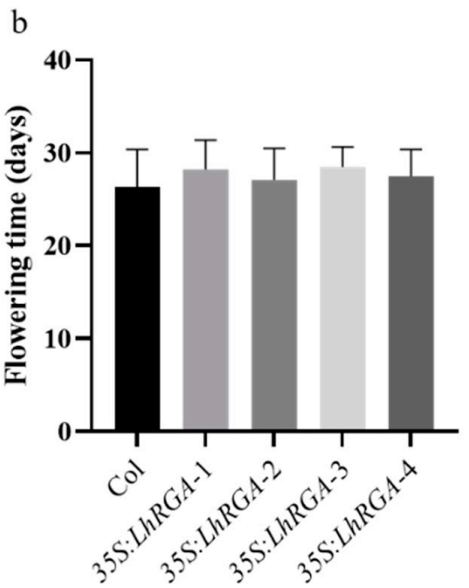

Col

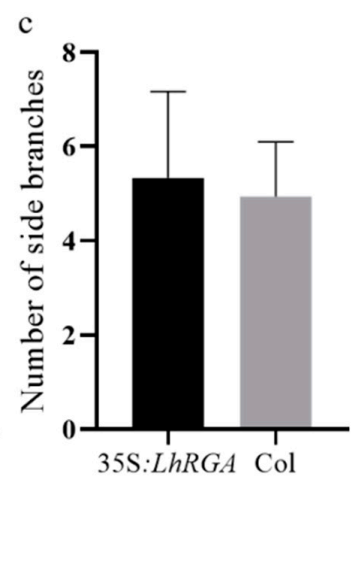

d

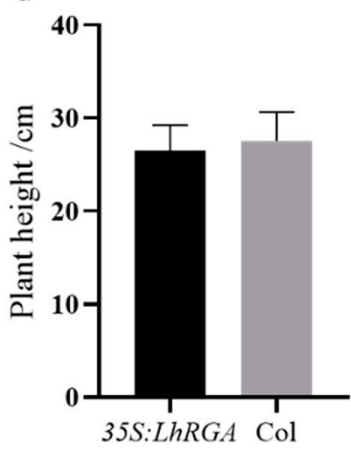

Figure 6. Morphological phenotypes of Arabidopsis overexpressing LhRGA. (a) Phenotypes of 30-day-old wild-type and transgenic plants. Scale bars $=2 \mathrm{~cm} \mathrm{(b)} \mathrm{Measurement} \mathrm{of} \mathrm{flowering} \mathrm{time} \mathrm{in}$ the wild-type and transgenic lines (a). Error bars: \pm SD. (c) Number of side branches in the wild-type and transgenic lines (a). Error bars: \pm SD. (d) Measurement of plant height branches in the wild-type and transgenic lines (a). Error bars: \pm SD.

As we had seen that LhSLRL may shorten plant roots in the Arabidopsis (Figure 5f,g), we wondered whether LhRGA might also affect root development. To study this, we designed an experiment using different concentrations of $\mathrm{GA}_{3}$ to treat T3 LhRGA transgenic plants and wild-type plants. We found that low concentrations of $\mathrm{GA}_{3}$ were able to promote root elongation of both wild-type and transgenic plants (Figure 8a-f). T-test analysis showed that the root length of wild-type was not significantly different from transgenic plants in the absence of $\mathrm{GA}_{3}$; however, under treatment with different concentrations of $\mathrm{GA}_{3}$, the root length of transgenic plants was significantly shorter than 
wild-type plants, with the strongest effect being observed at a $\mathrm{GA}_{3}$ concentration of $0.5 \mu \mathrm{M}$ (Figure $8 \mathrm{f}$ ). Taken together, these results show that $L h R G A$ overexpression also leads to shorter roots and regulates root development. However, its effect is only apparent upon $\mathrm{GA}_{3}$ treatment.
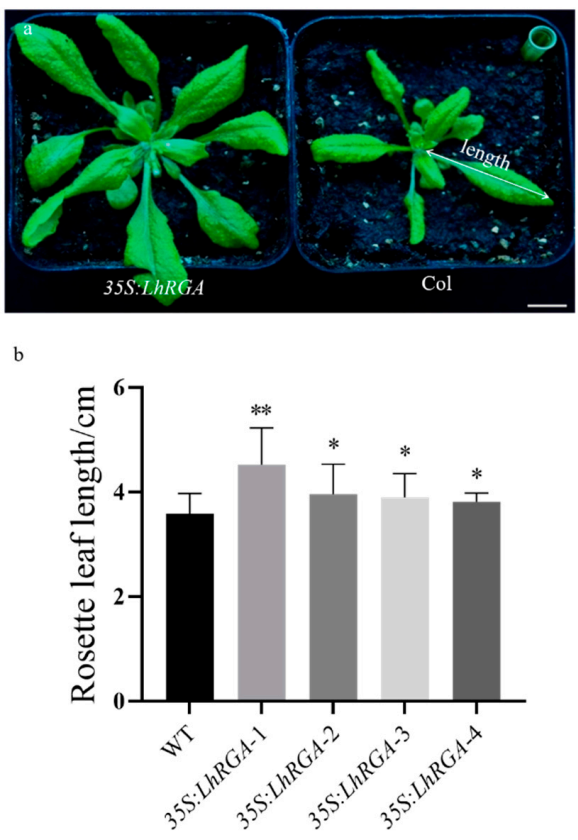

Figure 7. Morphological phenotypes of Arabidopsis overexpressing LhRGA. (a) Phenotypes of 28-day-old wild-type and transgenic plants. Scale bar $=1 \mathrm{~cm}$. (b) Measurement of rosette leaf length in the wild-type and transgenic lines (a). Error bars: \pm SD. ${ }^{*} p<0.05 ;{ }^{* *} p<0.01$.
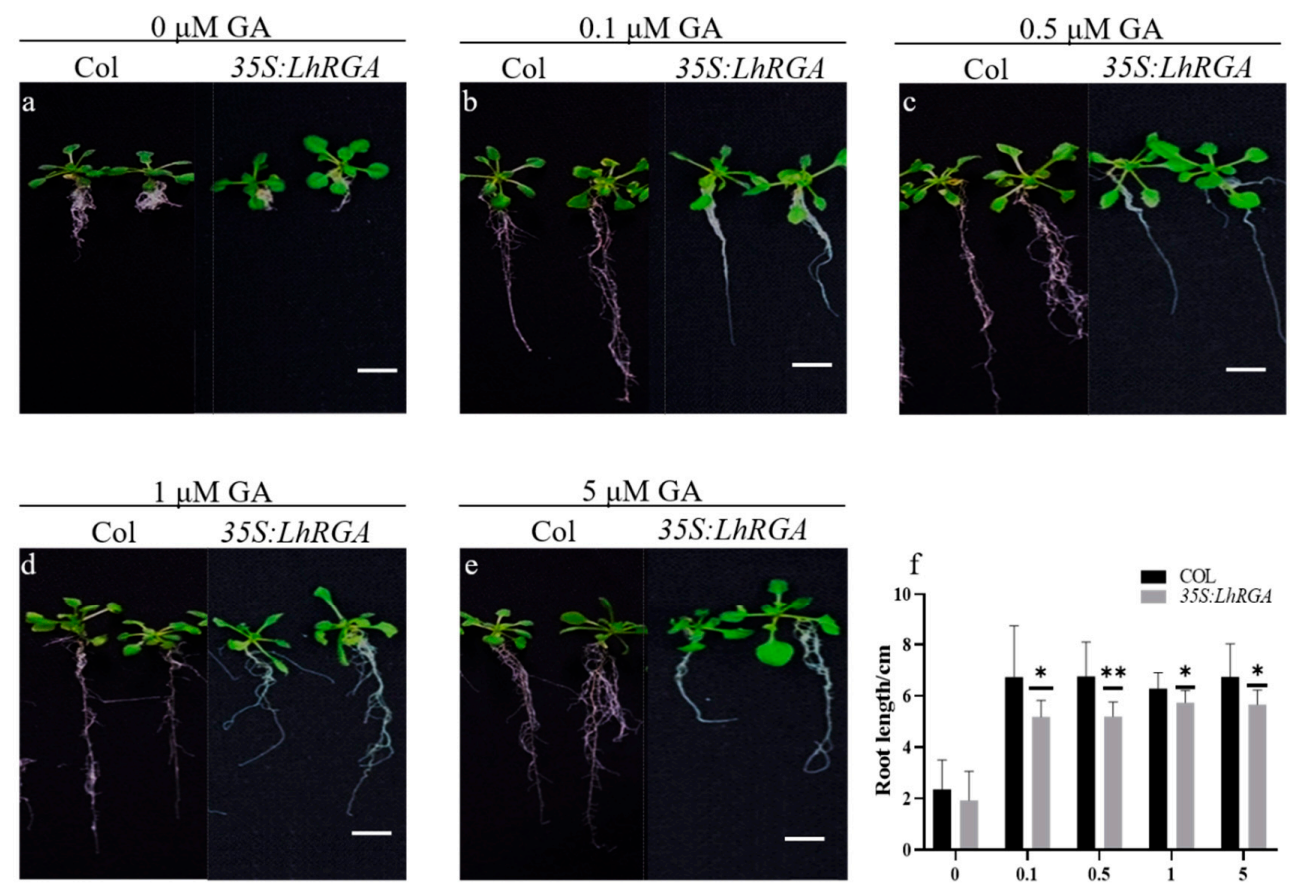

Figure 8. Morphological phenotypes of Arabidopsis overexpressing LhRGA after GA treatment. (a-e) Phenotypes of 15-day-old wild-type and transgenic plants treated with different concentrations of GA. Scale bar $=1 \mathrm{~cm}$. (f) Measurement of root length in the wild-type and transgenic lines (a-e). Error bars: \pm SD. ${ }^{*} p<0.05 ;{ }^{* *} p<0.01$. 


\section{Discussion}

In recent years, a large number of genes encoding DELLA proteins, a key regulator of the gibberellin signaling pathway, have been identified in poplar (Populus delotides $\times$ P. euramericana cv. 'Nanlin895') [44] and breadfruit (Artocarpus altilis cv. Cannonball) [45] and were subsequently isolated and cloned from other timber species. Although cloning of related genes in Liriodendron chinense (Hemsl.) Sarg. has been reported [46], the functionality of these genes has not yet been verified. Here, we successfully isolated two genes encoding DELLA proteins from Liriodendron hybrids. Liriodendron is a tertiary glacial relic plant, an ancient angiosperm with a history of more than 100 million years. However, the DELLA proteins of Liriodendron hybrids are strongly conserved, and have a similar number and arrangement of motifs as the DELLA proteins of other plants. This indicates that the Liriodendron hybrids DELLA proteins may have similar functions to those of other plants. However, compared with the DELLA protein of $A$. thaliana, the DELLA protein of Liriodendron hybrids lacks several motifs, suggesting that they may have different functions. More importantly, the DELLA domain of LhSLRL is significantly different from the same domain in DELLA proteins of other plants. The DELLA motif conserved in the DELLA domain of most plants is "DELLAALGYKV", whereas the DELLA motif in LhSLRL is "DGLLAGAGYHI". Single-base variation in the DELLA domain will cause plants to appear unresponsive to GA and show dwarfism. The $\sin 1 d$ dwarf mutant in barley is due to mutation of the conserved DELLALG motif in the DELLA domain to "DELLALE" [21]. In Arabidopsis overexpressing LhSLRL, there are significant differences in plant height, number of side branches, flowering time, and root length compared with the wild-type. However, compared with the wild-type, overexpressing LhRGA in Arabidopsis, only caused significant differences in rosette leaves, as well as the root length after $\mathrm{GA}_{3}$ treatment.

For a long time, it has been found that GAs can control branching, as evidenced by all GA-deficient mutants showing increased branching compared to wild-type [47]. The number of stem collaterals of Arabidopsis overexpressing LhSLRL was significantly higher than that of the wild-type, which is similar to the phenotype of GA-deficient mutants. This may be because the plant endogenous GAs are unable to degrade the overexpressed LhSLRL. Another possibility is that LhSLRL causes this phenotype through interaction with the plant hormone strigolactone (SL). SL is a terpene lactone, which was found to inhibit the growth of axillary buds. Studies have found that rice DELLA SLR1 can interact with D14 in an SL-dependent manner and regulate downstream SL signaling [48], thereby controlling the growth of axillary buds.

Recent studies have shown that GA enhances the anisotropy of leaf swelling [49]. Excessive GA makes the leaves narrow and long, whereas the GA-deficient dwarf3 mutant has short and wide leaves. The leaves of Arabidopsis overexpressing $L h R G A$ are long, which is different from the results of existing studies. This may be caused by divergence of extra motifs in LhRGA, or it may depend on additional factors being at play. In addition to the GA-DELLA module, there are five other modules that are important for the determination of Arabidopsis leaf size: ubiquitin receptor DA1-ENHANCER OF DA1 (EOD1), GROWTH REGULATING FACTOR (GRF)-GRF-INTERACTING FACTOR (GIF), SWITCH/SUCROSE NON-FERMENTING (SWI/SNF), KLU, and PEAPOD (PPD). These six modules form a complex regulatory network that regulates the size of Arabidopsis leaves [50]. The phenotypic effects observed when individual members of different modules are incorrectly expressed may also be balanced at the leaf level [51-53]. This may be the reason why the leaves of Arabidopsis overexpressing $L h R G A$ are long.

GAs can stimulate root elongation. Different concentrations of GA were applied to Arabidopsis overexpressing LhRGA, and it was found that the roots of $A$. thaliana had obvious elongation, but not as much as the wild-type. However, the effect of GAs on the formation of lateral and adventitious roots is still controversial. Early studies have shown that in some cases, exogenously administered GAs can inhibit this process [54], whereas in other cases it can promote this process [55]. In this study, both the lateral roots of Arabidopsis overexpressing LhSLRL and overexpressing LhRGA were significantly reduced, which was the opposite of the phenotype of the DELLA-deficient Populus 
tree [56]. The results showed that in Liriodendron hybrids, LhDELLA may inhibit the formation of lateral roots and adventitious roots, while GA promotes the formation of lateral roots and adventitious roots.

\section{Conclusions}

Overexpression of LhSLRL and LhRGA in Arabidopsis shows that both genes have remarkable effects on the vegetative growth of transgenic plants, but their functions may be different. Our results provide a preliminary understanding of LhDELLA function, supplying an important basis for further understanding of the mechanism of GA signal transduction and its role in the growth and development of Liriodendron hybrids.

Supplementary Materials: The following are available online at http://www.mdpi.com/1999-4907/11/12/1363/s1, Figure S1: Morphological phenotypes of tobacco overexpressing LhSLRL, Figure S2: Morphological phenotypes of Arabidopsis overexpressing LhRGA, Figure S3: Sampling method, Figure S4: PCR testing for Arabidopsis overexpressing LhSLRL, Figure S5: PCR testing for Arabidopsis overexpressing LhRGA, Figure S6: RT-PCR analysis of wild types and Arabidopsis/tobacco overexpressing LhDELLAs, Figure S7: PCR testing for tobacco overexpressing LhSLRL, Figure S8: The thermal profile of qRT-PCR, Table S1: DELLA proteins of different species, Table S2: Primers used for gene cloning, Table S3: Primers used for qRT-PCR, RT-PCR, and PCR testing, Table S4: Identity matrix for LhSLRL/LhRGA and five DELLA proteins in Arabidopsis.

Author Contributions: Y.L. (Yang Liu) and P.W. designed the experiments, prepared samples and drafted the manuscript. Y.L. (Yang Liu) carried out the bio-informatics analyses. Y.L. (Yang Liu), P.W., S.Y., X.L., L.L. and X.C. performed the experiments. Y.L. (Ye Lu) contributed to the reagents and performed experiments. Z.H. participated in directing the study. J.S. participated in the design of this project. J.C., as the corresponding author, provided the idea and designed the framework of this study, and modified the manuscript. All authors contributed to the final manuscript. All authors have read and agreed to the published version of the manuscript.

Funding: This work was supported by the Key Research and Development Plan of Jiangsu Province (BE2017376), the Nature Science Foundation of China (32071784, 31770715), the Qinglan Project of Jiangsu Province, the Distinguished Professor Project of Jiangsu province and the Priority Academic Program Development of Jiangsu Higher Education Institutions (PAPD).

Conflicts of Interest: The authors declare no competing interest.

\section{References}

1. Sun, T.-P. The Molecular Mechanism and Evolution of the GA-GID1-DELLA Signaling Module in Plants. Curr. Biol. 2011, 21, R338-R345. [CrossRef] [PubMed]

2. Daviere, J.M.; Achard, P. Gibberellin signaling in plants. Development 2013, 140, 1147-1151. [PubMed]

3. Ikeda, A.; Ueguchi-Tanaka, M.; Sonoda, Y.; Kitano, H.; Koshioka, M.; Futsuhara, Y.; Matsuoka, M.; Yamaguchi, J. Slender rice, a constitutive gibberellin response mutant, is caused by a null mutation of the SLR1 gene, an ortholog of the height-regulating gene GAI/RGA/RHT/D8. Plant Cell 2001, 13, 999-1010. [CrossRef] [PubMed]

4. Peng, J.; Carol, P.; Richards, D.E.; King, K.E.; Cowling, R.J.; Murphy, G.P.; Harberd, N.P. The Arabidopsis GAI gene defines a signaling pathway that negatively regulates gibberellin responses. Genes Dev. 1997, 11, 3194-3205. [CrossRef] [PubMed]

5. Ueguchi-Tanaka, M.; Ashikari, M.; Nakajima, M.; Itoh, H.; Katoh, E.; Kobayashi, M.; Chow, T.Y.; Hsing, Y.I.; Kitano, H.; Yamaguchi, I.; et al. Gibberellin insensitive DWARF1 encodes a soluble receptor for gibberellin. Nature 2005, 437, 693-698. [CrossRef] [PubMed]

6. Willige, B.C.; Ghosh, S.; Nill, C.; Zourelidou, M.; Dohmann, E.M.; Maier, A.; Schwechheimer, C. The DELLA Domain of GA INSENSITIVE Mediates the Interaction with the GA INSENSITIVE DWARF1A Gibberellin Receptor of Arabidopsis. Plant Cell 2007, 19, 1209-1220. [CrossRef]

7. Nakajima, M.; Shimada, A.; Takashi, Y.; Kim, Y.C.; Park, S.H.; Ueguchi-Tanaka, M.; Suzuki, H.; Katoh, E.; Iuchi, S.; Kobayashi, M.; et al. Identification and characterization of Arabidopsis gibberellin receptors. Plant J. 2006, 46, 880-889. [CrossRef]

8. Ariizumi, T.; Lawrence, P.K.; Steber, C.M. The Role of Two F-Box Proteins, SLEEPY1 and SNEEZY, in Arabidopsis Gibberellin Signaling. Plant Physiol. 2010, 155, 765-775. [CrossRef] 
9. Gomi, K.; Sasaki, A.; Itoh, H.; Ueguchi-Tanaka, M.; Ashikari, M.; Kitano, H.; Matsuoka, M. GID2, an F-box subunit of the SCF E3 complex, specifically interacts with phosphorylated SLR1 protein and regulates the gibberellin-dependent degradation of SLR1 in rice. Plant J. 2004, 37, 626-634. [CrossRef]

10. Vera-Sirera, F.; Gomez, M.D.; Perez-Amador, M.A. DELLA Proteins, a Group of GRAS Transcription Regulators that Mediate Gibberellin Signaling. In Plant Transcription Factors; Gonzalez, D.H., Ed.; Academic Press: Cambridge, MA, USA, 2016; pp. 313-328.

11. Wen, C.-K.; Chang, C. Arabidopsis RGL1 Encodes a Negative Regulator of Gibberellin Responses. Plant Cell 2002, 14, 87-100. [CrossRef]

12. Peng, J.; Richards, D.E.; Hartley, N.M.; Murphy, G.P.; Devos, K.M.; Flintham, J.E.; Beales, J.; Fish, L.J.; Worland, A.J.; Pelica, F.; et al. 'Green revolution' genes encode mutant gibberellin response modulators. Nature 1999, 400, 256-261. [CrossRef] [PubMed]

13. Zhao, B.; Li, H.; Li, J.; Wang, B.; Dai, C.; Wang, J.; Liu, K. Brassica napus DS-3, encoding a DELLA protein, negatively regulates stem elongation through gibberellin signaling pathway. Theor. Appl. Genet. 2017, 130, 727-741. [CrossRef] [PubMed]

14. Li, W.; Zhang, J.; Sun, H.; Wang, S.; Chen, K.; Liu, Y.; Li, H.; Ma, Y.; Zhang, Z. FveRGA1, encoding a DELLA protein, negatively regulates runner production in Fragaria vesca. Planta 2017, 247, 941-951. [CrossRef] [PubMed]

15. Silverstone, A.L.; Ciampaglio, C.N.; Sun, T. The Arabidopsis RGA gene encodes a transcriptional regulator repressing the gibberellin signal transduction pathway. Plant Cell 1998, 10, 155-169. [CrossRef] [PubMed]

16. Dill, A.; Jung, H.-S.; Sun, T.-P. The DELLA motif is essential for gibberellin-induced degradation of RGA. Proc. Natl. Acad. Sci. USA 2001, 98, 14162-14167. [CrossRef] [PubMed]

17. Lee, K.P.; Piskurewicz, U.; Turečková, V.; Strnad, M.; Lopez-Molina, L. A seed coat bedding assay shows that RGL2-dependent release of abscisic acid by the endosperm controls embryo growth in Arabidopsis dormant seeds. Proc. Natl. Acad. Sci. USA 2010, 107, 19108-19113. [CrossRef]

18. Wild, M.; Davière, J.-M.; Cheminant, S.; Regnault, T.; Baumberger, N.; Heintz, D.; Baltz, R.; Genschik, P.; Achard, P. The Arabidopsis DELLA RGA-LIKE3 Is a Direct Target of MYC2 and Modulates Jasmonate Signaling Responses. Plant Cell 2012, 24, 3307-3319. [CrossRef]

19. Hernández-García, J.; Briones-Moreno, A.; Dumas, R.; Blázquez, M.A. Origin of Gibberellin-Dependent Transcriptional Regulation by Molecular Exploitation of a Transactivation Domain in DELLA Proteins. Mol. Biol. Evol. 2019, 36, 908-918. [CrossRef]

20. Li, S.; Zhao, Y.; Zhao, Z.; Wu, X.; Sun, L.; Liu, Q.; Wu, Y. Crystal Structure of the GRAS Domain of SCARECROW-LIKE7 in Oryza sativa. Plant Cell 2016, 28, 1025-1034. [CrossRef]

21. Chandler, P.M.; Marion-Poll, A.; Ellis, M.; Gubler, F. Mutants at the Slender1 Locus of Barley cv Himalaya. Molecular and Physiological Characterization. Plant Physiol. 2002, 129, 181-190. [CrossRef]

22. Luo, A.; Qian, Q.; Yin, H.; Liu, X.; Yin, C.; Lan, Y.; Tang, J.; Tang, Z.; Cao, S.; Wang, X.; et al. EUI1, Encoding a Putative Cytochrome P450 Monooxygenase, Regulates Internode Elongation by Modulating Gibberellin Responses in Rice. Plant Cell Physiol. 2006, 47, 181-191. [CrossRef] [PubMed]

23. La Rosa, N.M.-D.; Sotillo, B.; Miskolczi, P.; Gibbs, D.J.; Vicente, J.; Carbonero, P.; Oñate-Sánchez, L.; Holdsworth, M.J.; Bhalerao, R.; Alabadí, D.; et al. Large-Scale Identification of Gibberellin-Related Transcription Factors Defines Group VII ethylene response factors as Functional della partners. Plant Physiol. 2014, 166, 1022-1032. [CrossRef] [PubMed]

24. Lantzouni, O.; Alkofer, A.; Falter-Braun, P.; Schwechheimer, C. Growth-regulating factors Interact with DELLAs and Regulate Growth in Cold Stress. Plant Cell 2020, 32, 1018-1034. [CrossRef] [PubMed]

25. de Lucas, M.; Daviere, J.M.; Rodriguez-Falcon, M.; Pontin, M.; Iglesias-Pedraz, J.M.; Lorrain, S.; Fankhauser, C.; Blazquez, M.A.; Titarenko, E.; Prat, S. A molecular framework for light and gibberellin control of cell elongation. Nature 2008, 451, 480-484. [CrossRef]

26. Gallego-Bartolomé, J.; Minguet, E.G.; Grau-Enguix, F.; Abbas, M.; Locascio, A.; Thomas, S.G.; Alabadí, D.; Blázquez, M.A. Molecular mechanism for the interaction between gibberellin and brassinosteroid signaling pathways in Arabidopsis. Proc. Natl. Acad. Sci. USA 2012, 109, 13446-13451. [CrossRef]

27. La Rosa, N.M.; Pfeiffer, A.; Hill, K.; Locascio, A.; Bhalerao, R.P.; Miskolczi, P.C.; Grønlund, A.L.; Wanchoo-Kohli, A.; Thomas, S.G.; Bennett, M.J.; et al. Genome Wide Binding Site Analysis Reveals Transcriptional Coactivation of Cytokinin-Responsive Genes by DELLA Proteins. PLoS Genet. 2015, 11, e1005337. [CrossRef] 
28. Fukazawa, J.; Teramura, H.; Murakoshi, S.; Nasuno, K.; Nishida, N.; Ito, T.; Yoshida, M.; Kamiya, Y.; Yamaguchi, S.; Takahashi, Y. DELLAs Function as Coactivators of GAI-ASSOCIATED FACTOR1 in Regulation of Gibberellin Homeostasis and Signaling in Arabidopsis. Plant Cell 2014, 26, 2920-2938. [CrossRef]

29. Locascio, A.; Blázquez, M.A.; Alabadí, D. Genomic Analysis of DELLA Protein Activity. Plant Cell Physiol. 2013, 54, 1229-1237. [CrossRef]

30. Thomas, S.G. Novel Rht-1 dwarfing genes: Tools for wheat breeding and dissecting the function of DELLA proteins. J. Exp. Bot. 2017, 68, 354-358. [CrossRef]

31. Liu, M.; Huang, L.; Ma, Z.; Sun, W.; Wu, Q.; Tang, Z.; Bu, T.; Li, C.; Chen, H. Genome-wide identification, expression analysis and functional study of the GRAS gene family in Tartary buckwheat (Fagopyrum tataricum). BMC Plant Biol. 2019, 19, 1-17. [CrossRef]

32. Xie, Y.; Tan, H.; Ma, Z.; Huang, J. DELLA Proteins Promote Anthocyanin Biosynthesis via Sequestering MYBL2 and JAZ Suppressors of the MYB/bHLH/WD40 Complex in Arabidopsis thaliana. Mol. Plant 2016, 9, 711-721. [CrossRef] [PubMed]

33. Achard, P. Integration of Plant Responses to Environmentally Activated Phytohormonal Signals. Science 2006, 311, 91-94. [CrossRef] [PubMed]

34. Chen, C.; Chen, H.; He, Y.; Xia, R. TBtools, a Toolkit for Biologists integrating various HTS-data handling tools with a user-friendly interface. bioRxiv 2018. [CrossRef]

35. Jones, D.T.; Taylor, W.R.; Thornton, J.M. The rapid generation of mutation data matrices from protein sequences. Bioinformatics 1992, 8, 275-282. [CrossRef] [PubMed]

36. Kumar, S.; Stecher, G.; Li, M.; Knyaz, C.; Tamura, K. MEGA X: Molecular Evolutionary Genetics Analysis across Computing Platforms. Mol. Biol. Evol. 2018, 35, 1547-1549. [CrossRef] [PubMed]

37. Lloyd, A.M.; Barnason, A.R.; Rogers, S.G.; Byrne, M.C.; Fraley, R.T.; Horsch, R.B. Transformation of Arabidopsis thaliana with Agrobacterium tumefaciens. Science 1986, 234, 464-466. [CrossRef]

38. Clough, S.J.; Bent, A.F. Floral dip: A simplified method for Agrobacterium-mediated transformation of Arabidopsis thaliana: Floral dip transformation of Arabidopsis. Plant J. Cell Mol. Biol. 1998, 16, 735-743. [CrossRef]

39. Li, T.; Chen, J.; Qiu, S.; Zhang, Y.; Wang, P.; Yang, L.; Lu, Y.; Shi, J. Deep Sequencing and Microarray Hybridization Identify Conserved and Species-Specific MicroRNAs during Somatic Embryogenesis in Hybrid Yellow Poplar. PLoS ONE 2012, 7, e43451. [CrossRef]

40. Livak, K.J.; Schmittgen, T.D. Analysis of relative gene expression data using real-time quantitative PCR and the 2(-Delta Delta C(T)) Method. Methods 2001, 25, 402-408. [CrossRef]

41. Chen, J.; Yang, G.-X.; Ding, Q.; Xia, T.-S.; Shi, J.; Jia, A.-Q. In vitro tumor cytotoxic activities of extracts from three Liriodendron plants. Pak. J. Pharm. Sci. 2013, 26, 233-237.

42. Chen, J.; Hao, Z.; Guang, X.; Zhao, C.; Wang, P.; Xue, L.; Zhu, Q.; Yang, L.; Sheng, Y.; Zhou, Y.; et al. Liriodendron genome sheds light on angiosperm phylogeny and species-pair differentiation. Nat. Plants 2019, 5, 18-25. [CrossRef] [PubMed]

43. Davière, J.-M.; Achard, P. A Pivotal Role of DELLAs in Regulating Multiple Hormone Signals. Mol. Plant 2016, 9, 10-20. [CrossRef]

44. Liu, S.; Xuan, L.; Xu, L.-A.; Huang, M.; Xu, M. Molecular cloning, expression analysis and subcellular localization of four DELLA genes from hybrid poplar. SpringerPlus 2016, 5, 1-8. [CrossRef] [PubMed]

45. Zhou, Y.; Underhill, S.J.R. Breadfruit (Artocarpus altilis) DELLA genes: Gibberellin-regulated stem elongation and response to high salinity and drought. Plant Growth Regul. 2017, 83, 375-383. [CrossRef]

46. Nie, Z.-L.; Wen, J.; Azuma, H.; Qiu, Y.-L.; Sun, H.; Meng, Y.; Sun, W.-B.; Zimmer, E.A. Phylogenetic and biogeographic complexity of Magnoliaceae in the Northern Hemisphere inferred from three nuclear data sets. Mol. Phylogenet. Evol. 2008, 48, 1027-1040. [CrossRef] [PubMed]

47. Lo, S.-F.; Yang, S.-Y.; Chen, K.-T.; Hsing, Y.-I.; Zeevaart, J.A.; Chen, L.-J.; Yu, S.-M. A Novel Class of Gibberellin 2-Oxidases Control Semidwarfism, Tillering, and Root Development in Rice. Plant Cell 2008, 20, 2603-2618. [CrossRef] [PubMed]

48. Nakamura, H.; Xue, Y.; Miyakawa, T.; Hou, F.; Qin, H.; Fukui, K.; Shi, X.; Ito, E.; Ito, S.; Park, S.; et al. Molecular mechanism of strigolactone perception by DWARF14. Nat. Commun. 2013, 4, 2613. [CrossRef]

49. Sprangers, K.; Thys, S.; Van Dusschoten, D.; Beemster, G.T. Gibberellin Enhances the Anisotropy of Cell Expansion in the Growth Zone of the Maize Leaf. Front. Plant Sci. 2020, 11, 1163. [CrossRef] 
50. Vercruysse, J.; Baekelandt, A.; Gonzalez, N.; Inzé, D. Molecular networks regulating cell division during Arabidopsis leaf growth. J. Exp. Bot. 2020, 71, 2365-2378. [CrossRef]

51. Gonzalez, N.; Vanhaeren, H.; Inzé, D. Leaf size control: Complex coordination of cell division and expansion. Trends Plant Sci. 2012, 17, 332-340. [CrossRef]

52. Zhang, Y.; Du, L.; Xu, R.; Cui, R.; Hao, J.; Sun, C.; Li, Y. Transcription Factors SOD7/NGAL2 and DPA4/NGAL3 Act Redundantly to Regulate Seed Size by Directly Repressing KLU Expression in Arabidopsis thaliana. Plant Cell 2015, 27, 620-632. [CrossRef] [PubMed]

53. Wang, Z.; Li, N.; Jiang, S.; Gonzalez, N.; Huang, X.; Wang, Y.; Inzé, D.; Li, Y. SCFSAP controls organ size by targeting PPD proteins for degradation in Arabidopsis thaliana. Nat. Commun. 2016, 7, 11192. [CrossRef] [PubMed]

54. Brian, P.W.; Hemming, H.G.; Lowe, D. Inhibition of Rooting of Cuttings by Gibberellic Acid: With one Figure in the Text. Ann. Bot. 1960, 24, 407-419. [CrossRef]

55. Nanda, K.K.; Anand, V.K.; Chibbar, R.N. The promotive effect of gibberellic acid on the production of adventitious roots on stem cuttings of Ipomoea fistulosa. Planta 1972, 105, 360-363. [CrossRef]

56. Busov, V.B.; Meilan, R.; Pearce, D.W.; Rood, S.B.; Ma, C.; Tschaplinski, T.J.; Strauss, S.H. Transgenic modification of gai or rgl1 causes dwarfing and alters gibberellins, root growth, and metabolite profiles in Populus. Planta 2006, 224, 288-299. [CrossRef]

Publisher's Note: MDPI stays neutral with regard to jurisdictional claims in published maps and institutional affiliations. 